\title{
Correction to: Care Ethics and Nursing Practice
}

\author{
Ann Gallagher
}

\section{Correction to:}

Chapter 5 in: P. A. Scott, Key Concepts and Issues in Nursing Ethics, https://doi.org/10.1007/978-3-319-49250-6_5

Author noticed errors in her affiliation after publication of the book.

The affiliation has been updated to read:

\author{
A. Gallagher \\ International Care Ethics (ICE) Observatory, \\ School of Health Sciences, \\ Faculty of Health and Medical Sciences, \\ University of Surrey, \\ Guildford GU2 7XH, UK
}

The original version for this chapter can be found at https://doi.org/10.1007/978-3-319-49250-6_5

\footnotetext{
A. Gallagher $(\bowtie)$

International Care Ethics (ICE) Observatory, School of Health Sciences,

Faculty of Health and Medical Sciences, University of Surrey,

Guildford GU2 7XH, UK

e-mail: a.gallagher@surrey.ac.uk
} 